Article

\title{
Characterization of Two AGL6-Like Genes from a Chinese Endemic Woody Tree, Manglietia patungensis (Magnoliaceae) Provides Insight into Perianth Development and Evolution in Basal Angiosperms
}

\author{
Zhixiong Liu ${ }^{1, *}$, Kebin Zhang ${ }^{1}$, Laiyun Li ${ }^{1}$, Yue Fei ${ }^{1}$ and Faju Chen ${ }^{2}$ \\ College of Horticulture and Gardening, Yangtze University, Jingzhou 434025, China \\ 2 Key Laboratory of Three Gorges Regional Plant Genetics \& Germplasm Enhancement (CTGU), \\ China Three Gorges University, Yichang 443002, China \\ * Correspondence: zxliu@yangtzeu.edu.cn; Tel.: +86-716-8066260
}

Received: 23 May 2019; Accepted: 7 August 2019; Published: 8 August 2019

\begin{abstract}
Manglietia patungensis (Magnoliaceae) exhibits radially symmetric flowers with perianth consisting of three separate sepaloid tepals in whorl 1 and six petaloid tepals in the inner two whorls, which shows an obvious difference from flowers of most Magnoliaceae species that contain three uniform petaloid tepals whorls, and make it an excellent model for understanding perianth morphology differentiation during early flower evolution. Here, two AGL6 orthologs, MapaAGL6-1 and MapaAGL6-2, were isolated from M. patungensis. Sequence alignment and phylogenetic analyses grouped both genes into the AGL6 lineage. MapaAGL6-1 is expressed only in the perianth whorls, while MapaAGL6-2 is strongly expressed in the perianth whorls but is lowly expressed in gynoecium. Furthermore, ectopic expression of MapaAGL6-1 results in strong complementation phenotypes in the Arabidopsis ap 1-10 flower and production of normal floral organs in four floral whorls only with the petal number reduced in whorl 2, while ectopic expression of MapaAGL6-2 only results in petals partly rescued but failing to terminate carpelloid development in Arabidopsis ap1-10 mutant. In addition, the daughter lines generated from a cross between 35S::MapaAGL6-1 transgenic plants showing strong phenotypes and 35S::MapaAGL6-2 transgenic plants showing phenotypic changes produce normal flowers. Our results suggest that MapaAGL6-1 is a reasonable A-function gene controlling perianth identity in Magnoliaceae, which infers from its expression region and complementation phenotypes in Arabidopsis ap1 mutant, while MapaAGL6-2 is mainly involved in petaloid tepal development. Our data also provide a new clue to uncover the perianth development and early evolution in basal angiosperms.
\end{abstract}

Keywords: AGAMOUS-LIKE6; floral development; Manglietia patungensis; MADS-box gene; perianth

\section{Introduction}

The long-established ABCE model has provided a detailed picture of how perfect floral organs are formed in the core eudicot model systems Arabidopsis and Antirrhinum [1-3]. According to the model, the expressions of A-function genes are restricted to the first and second whorls for specifying perianth (sepal and petal) identity by the C-class genes [3,4]. APETALA1 (AP1) and AP2 reportedly confer A function in Arabidopsis. However, the AP1 ortholog from Antirrhinum, SQUAMOSA (SQUA), does not have A-function, although the AP2 homologs, LIPLESS1 (LIP1) and LIP2, confer partial A-function by contributing to perianth identity [5]. Previous study has suggested that the A-function for AP1 may be derived in Arabidopsis and perhaps relative species in the rosid clade, but this function has not yet been demonstrated in any species other than Arabidopsis [6]. Notably, the AGAMOUS-LIKE6 (AGL6) 
lineage member, NdAGL6 from a basal eudicot species Nigella damascena, has been identified to be an A-function gene [7]. Also, AGL6 homologs from other basal angiosperm species, such as Magnolia grandiflora and Amborella trichopoda, have been strongly expressed in tepals [8]. In addition, an AGL6 ortholog, AfimAGL6 from another basal angiosperm, Aristolochia fimbriata, has been observed to be exclusively expressed in flowers and capsules and to be involved in regulating sepal identity $[9,10]$. In monocots, the AGL6 ortholog, OsMADS6 from Oryza sativa, has been seen to be strongly expressed in paleas (sepal homologs), lodicules (petal homologs), carpels and receptacle, and regulated flower development of four whorls in rice [11,12]. Moreover, three AGL6-like genes have been found in orchids, including Erycina pusilla and Cymbidium goeringii, and has suggested subfunctionalization of the orchid AGL6 orthologs following gene duplication events [13,14]. For example, EpMADS4 from E. pusilla has been seen to be lowly expressed in the median sepal which has resulted in petaloid sepal appearance, while EpMADS5 from E. pusilla has been observed to be highly expressed in the lips and to be involved in lip development [13]. In addition, CgAGL6-1 from C. goeringii has been found to be strongly expressed in the sepals and petals and to be involved in promoting sepal/petal formation, while CgAGL6-3 from C. goeringii has been seen to be highly expressed in the lips and to be involved in lip development [14]. In asterid eudicots, AGL6 orthologs, such as SIAGL6 from Solanum lycopersicon and PhAGL6 from Petunia hybrida, have been found to be expressed in all floral organs $[15,16]$. However, SlAGL6 has been seen to be mainly expressed in sepals, petals, and carpels, but also to be involved in tomato sepal and petal development [15]. PhAGL6 has been seen to be mainly expressed in petals, anthers, and ovaries, and has been found to work together with SEPALLATA $(S E P)$ genes to regulate petal, anther, and ovary development [15,16]. Functional identifications of AGL6 orthologs from different clades of angiosperms species have suggested interesting examples of expression patterns and functional evolution.

Here, two AGL6 homolog mRNAs were isolated from Manglietia patungensis, a Chinese endemic woody tree that belongs to the family Magnoliaceae. M. patungensis produces perianth consisting of three whorls of three separate tepals, with the first whorl of three sepaloid tepals and the inner two whorls of six petaloid tepals (Figure 1), which exhibits obvious differences from typical Magnoliaceae flowers, which have three uniform petaloid tepals whorls that are highly similar to the putative ancestral angiosperm flower perianth [17], and make it an excellent model for understanding perianth morphology differentiation during early flower evolution. Sequence alignment and phylogenetic analyses grouped both AGL6 homologs, MapaAGL6-1 and MapaAGL6-2, into the AGL6 lineage. Expression analyses showed that MapaAGL6-1 and MapaAGL6-2 were mainly expressed in the development tepals. Ectopic expression of MapaAGL6-1 in Arabidopsis ap1-10 mutants produced flowers with sepaloid organs in whorl 1 and petaloid structures in whorl 2, while many of the 35S::MapaAGL6-2 transgenic Arabidopsis ap1-10 mutants produced flowers with the anthers converted into petaloid organs. Our data provide a new clue to uncovering the differentiation of sepaloid and petaloid tepals in basal angiosperms.

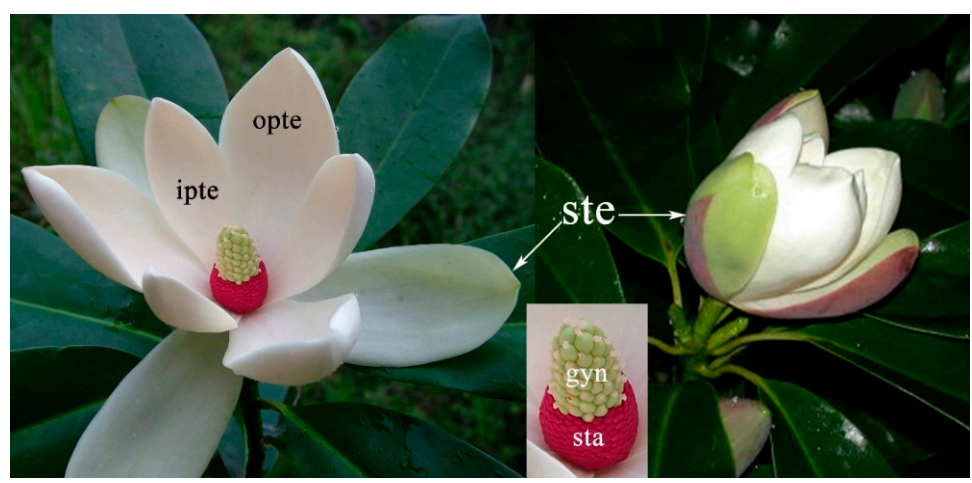

Figure 1. Flower of Manglietia patungensis. Shown are the sepaloid tepal (ste), outer petaloid tepal (opte), inner petaloid tepal (ipte), stamen (sta), and gynoecium (gyn). 


\section{Materials and Methods}

\subsection{Materials}

Flower buds at various developmental stages were collected from M. patungensis growing under natural conditions in the Three Gorges Botanical Garden, Yichang, Hubei Province. Juvenile leaves, sepaloid tepals, outer petaloid tepals, inner petaloid tepals, stamens, and gynoecia were sampled before anthesis, immediately frozen in liquid nitrogen, and stored at $-80^{\circ} \mathrm{C}$ until use. The Arabidopsis ap 1-10 mutant line (CS6230) in ecotype Landsberg background was obtained from the Arabidopsis Biological Resource Center, Ohio State University, Columbus, OH, USA.

\subsection{Isolation and Identification of AGL6 Homologs from M. patungensis}

Total RNAs were extracted from floral buds using an EASYspin Plant RNA Extraction Kit (Aidlab China) following the manufacturer's protocol. First-strand cDNA was synthesized from $2 \mu \mathrm{g}$ of total RNA using an oligo (dT)18 adaptor primer and M-MLV Reverse Transcriptase (TaKaRa, Japan). MapaAGL6-1 cDNA was isolated by PCR amplification using the forward primer MapaAGL6-1F (5'-GTCTAGAAATCATCTCGTACAAAAG-3') and the reverse primer MapaAGL6-1R (5'-GGAGCTCTACAAACCATATTGTGTG-3'). MapaAGL6-2 cDNA was isolated by PCR amplification using the forward primer MapaAGL6-2F (5'-GTCTAGAGAAAATCTGAGATGGGGAG-3') and the reverse primer MapaAGL6-2R (5'-GGAGCTCAAGTTTGGACCAGATTCATGC-3'). Primer design was based on the sequences of AGL6 homologs from close species, such as MawuAGL6-1 (Genbank accession numbers: KU860522.1) and MawuAGL6-2 (Genbank accession numbers: KU860523.1) from M. wufengensis, respectively. PCR was performed with a $3 \mathrm{~min} 94^{\circ} \mathrm{C}$ denaturation step, followed by 30 cycles of $30 \mathrm{~s}$ at $94{ }^{\circ} \mathrm{C}, 30 \mathrm{~s}$ annealing at $57^{\circ} \mathrm{C}$, a $45 \mathrm{~s}$ extension at $72{ }^{\circ} \mathrm{C}$, and then a final extension period of $10 \mathrm{~min}$.

\subsection{Sequence Alignments and Phylogenetic Analysis}

Deduced amino acid sequences of MapaAGL6-1 and MapaAGL6-2, and other MADS-box proteins from various angiosperm lineages were selected for phylogenetic trees from NCBI Genbank (Table S1). Phylogenetic trees were constructed with MEGA5.0 software using the maximum likelihood method with 1000 bootstrap replication [18]. Full-length amino acid sequences of AGL6-like proteins containing the MADS, I, $\mathrm{K}$ and $\mathrm{C}$ domains were aligned with BioEdit 7.0.9 software using the ClustalW program with default settings. Sequence data used were as follows: M. patungensis, MapaAGL6-1 (ATB53133.1), MapaAGL6-2 (ATB53135.1); A. thaliana, AGL6 (AEC10582.1), AGL13 (AEE80158.1) [19]; A. fimbriata, AfimAGL6 (ALV83432.1) [9]; Chimonanthus praecox, CpAGL6 (ACN88212.1) [20]; N. damascene, NdAGL6 (ALM95509.1) [5]; S. lycopersicum, SlAGL6 (NP_001348459.1) [21]; O. sativa, OsMADS6 (Q6EU39.1), OsMADS17 (ACX35552.1) [22].

\subsection{Expression Analysis of MapaAGL6-1 and MapaAGL6-2}

Juvenile leaves, four whorls of floral organs (sepaloid tepals, petaloid tepals, stamens, and gynoecium) from floral buds (27 $\mathrm{mm}$ in length) and five whorls of floral organs (sepaloid tepals, outer petaloid tepals, inner petaloid tepals, stamens, and gynoecium) from floral buds (40 mm in length) were collected from M. patungensis, respectively. Moreover, flower buds at various developmental stages were also sampled. Total RNAs were extracted from these organs and the first-strand cDNAs were subsequently synthesized using the protocol described above. Expressions of MapaAGL6-1 and MapaAGL6-2 were detected in these organs using quantitative real-time PCR (qPCR), respectively. Quantitative real-time PCR with three biological replicates was carried out according to Zhang et al. [23] with the gene-specific primers qMapaAGL6-1F (5'-ACTTATCGTCTTCTCCAGCC-3') and qMapaAGL6-1R (5' -ACCATCCCTGTGTATCACG-3') for MapaAGL6-1, as well as qMapaAGL6-2F (5'-GATGCTGAGGTCGCTCTTA-3') and qMapaAGL6-2R (5'-TCAACTTGGACACTTCCTGA-3') for MapaAGL6-2. Amplification of M. patungensis actin 
with specific primers qMpactinF (5'-AAGAACATCCCGTCCTCCTTACTG-3') and qMpactinR (5'-ACCGGAATCAAGCACAATACCTGT-3') was used as a normalization.

\subsection{Vector Construction and Arabidopsis Transformation}

Full-length MapaAGL6-1 and MapaAGL6-2 cDNAs in the sense orientation were cloned into binary vector pBI121 (BD Biosciences, Clontech) with Xba I and Sac I restriction enzymes under control of the CaMV35S promoter, respectively. The 35S::MapaAGL6-1 and 35S::MapaAGL6-2 constructs were transformed into homozygous ap1-10 Arabidopsis using the floral-dip method according to the protocol suggested by Clough and Bent via Agrobacterium tumefaciens strain GV3101, respectively [24]. Plasmid pBI121(negative control) was also transformed into homozygous ap1-10 Arabidopsis using the same protocol described above. Transgenic Arabidopsis seeds were selected and seedlings were cultivated according to Li et al. [25]. Moreover, transgenic A. thaliana lines were confirmed by PCR and qPCR as described above using the same gene-specific primers for MapaAGL6-1 and MapaAGL6-2, respectively. Amplification of the A. thaliana $\beta$-actin with the primers qactinF (5'-GATTTGGCATCACACTTTCTACAATG-3') and qactinR (5'-GTTCCACCACTGAGCACAATG-3') was used as a normalization. Transgenic homozygous mutant plants were analyzed after genotyping.

\subsection{Statistical Analysis}

All experiments were carried out with three biological replicates and data were expressed as mean \pm SD (standard deviation). Statistical significance was determined by $L S D$, and statistical significance was declared at $p$ value $\leq 0.01$ or 0.05 , respectively.

\section{Results}

\subsection{Cloning and Sequence Alignments of MapaAGL6-1 and MapaAGL6-2}

The MapaAGL6-1 cDNA is 822 bp with a 729 bp ORF (Open Reading Frame, ORF) encoding 242 amino acids (aa) (Genbank accession number: KX926430.1), whereas the MapaAGL6-2 cDNA is 833 bp with a 729 bp ORF encoding 242 aa (Genbank accession number: KX926432.1). Deduced proteins alignment and a phylogenetic tree grouped both the MapaAGL6-1 and MapaAGL6-2 into AGL6 lineage (Figure 2). Hence both genes were designated as MapaAGL6-1 (Manglietia patungensis AGAMOUS-like 6) and MapaAGL6-2, respectively. Conceptual translation shows that both proteins with 242 aa contain a 57 highly conserved MADS-box domain (1-57) at the N-terminal region, a 68 aa moderately conserved $\mathrm{K}$ domain (89-156) in the middle region, and an 86 aa variable $C$ domain (157-242), but with two highly conserved AGL6 I and II motifs (Figure 3) in the C-terminal region [26-28]. Moreover, both proteins consist of three putative amphipathic $\alpha$-helices referred to as the K1 (89-110), K2 (123-137), and K3 (145-177) subdomain with conserved hydrophobic amino acids at the a and d positions in the (abcdefg) $)_{n}$ heptad repeats [27]. However, both proteins show an $81.40 \%$ sequence identity. Two important residues of MapaAGL6-1 show difference with MapaAGL6-2 even in the key position with expected hydrophobic amino acids of the K1 and K2 subdomains (Figure 3). For example, Cys-100 in the K1 and Thr-137 in the K2 of MapaAGL6-1 correspond to Tyr-100 in the K1 and Ala-137 in the K2 of MapaAGL6-2, respectively. In addition, several amino acids residues in the K3 and AGL6 motifs II also display differences between MapaAGL6-1 and MapaAGL6-2. 


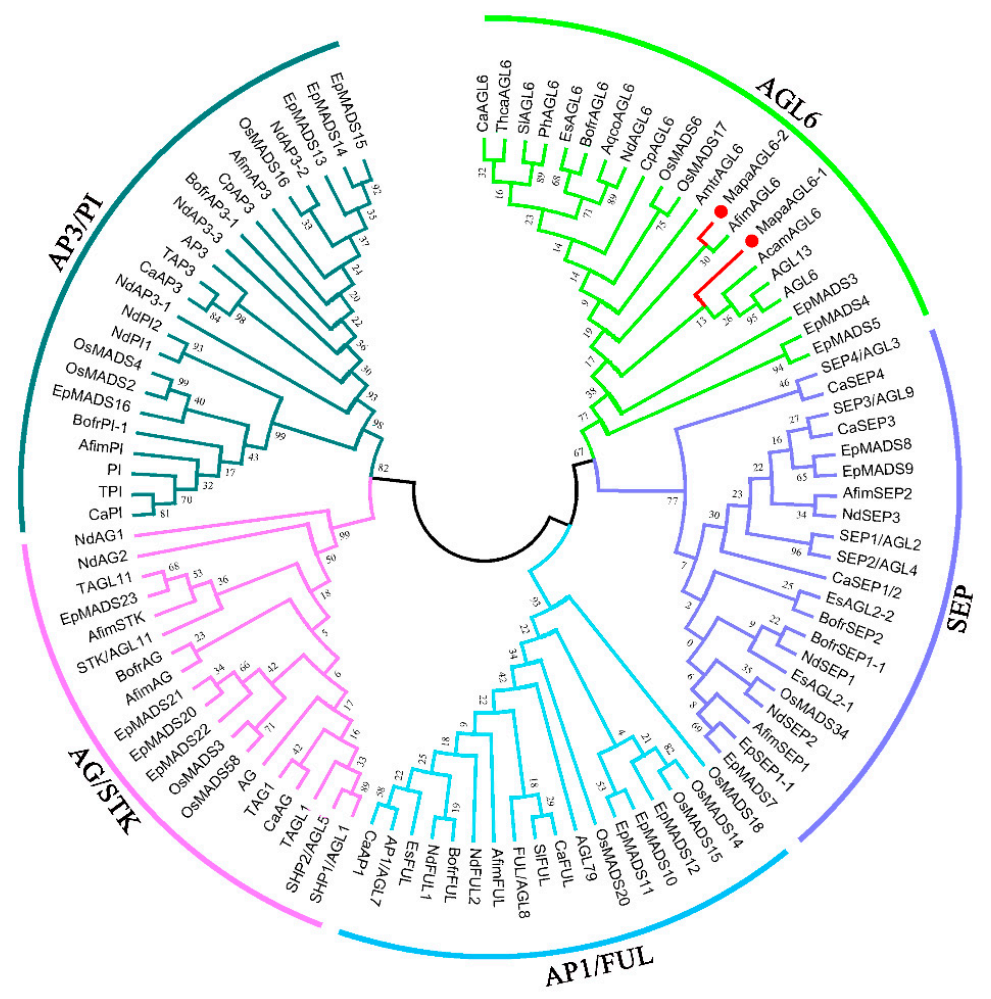

Figure 2. Phylogenetic analysis of MADS-box proteins. The tree was constructed with MEGA5.0 software using the maximum likelihood method. Numbers along the branches are bootstrap values from 1000 replications.

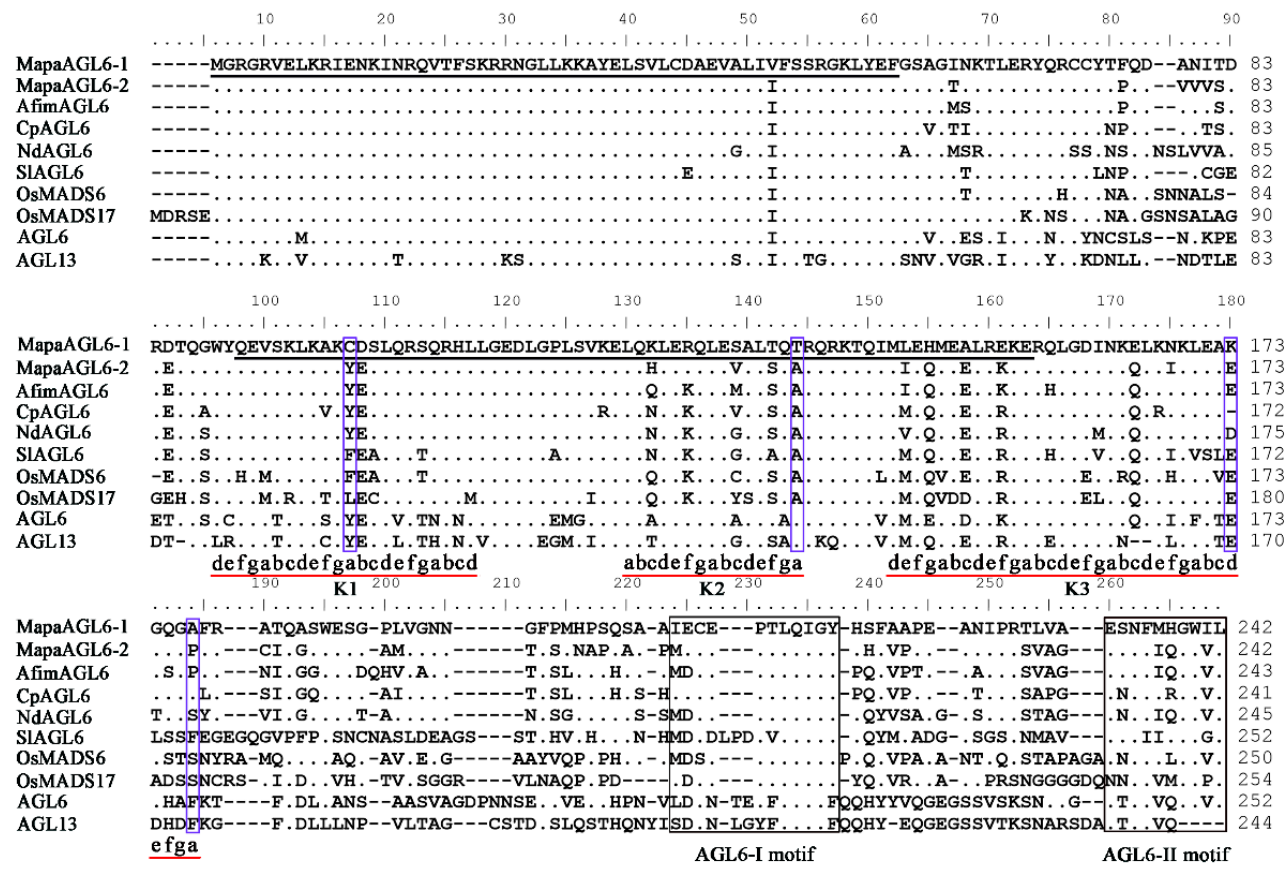

Figure 3. Sequence alignments of MapaAGL6-1 and MapaAGL6-2 with AGL6-like MADS-box proteins from other plants. The first underlined region represents the MADS domain and the second the $\mathrm{K}$ domain. The AGL6-I motif and AGL6-II motif in the C-terminal region are boxed. Amino acid residues identical to MapaAGL6-1 are indicated as dots. Dashes were introduced into the sequence to improve the alignment. Three heptad (abcdefg) $n$ repeats represented by k1, k2, and $\mathrm{k} 3$ subdomains that usually contain hydrophobic amino acids are present at positions a and $\mathrm{d}$ [27]. 


\subsection{Expression Analyses of MapaAGL6-1 and MapaAGL6-2 in M. patungensis}

MapaAGL6-1 and MapaAGL6-2 were mainly expressed in sepaloid tepals of $27 \mathrm{~mm}$ floral buds and both genes in the first whorl of floral organs were expressed significantly higher than that in other floral whorls. In addition, MapaAGL6-2 was also found in petaloid tepals and gynoecia for its low expression levels, whereas MapaAGL6-1 was only found for its low expression levels in petaloid tepals (Figure 4a). Moreover, with the floral bud increasing in length and the petaloid tepals developing, MapaAGL6-1 expression was extended inward into the inner petaloid tepals of $40 \mathrm{~mm}$ floral buds, while high levels of MapaAGL6-2 expression were also detected in the perianth whorls (Figure 4b). Furthermore, MapaAGL6-1 and MapaAGL6-2 expressions were also detected in $16 \mathrm{~mm}, 22 \mathrm{~mm}, 35 \mathrm{~mm}$, $38 \mathrm{~mm}$, and $52 \mathrm{~mm}$ floral buds, respectively. In addition, the expressions of both genes achieved high levels in $52 \mathrm{~mm}$ floral buds when the perianth rapidly enlarged in size (Figure 5).
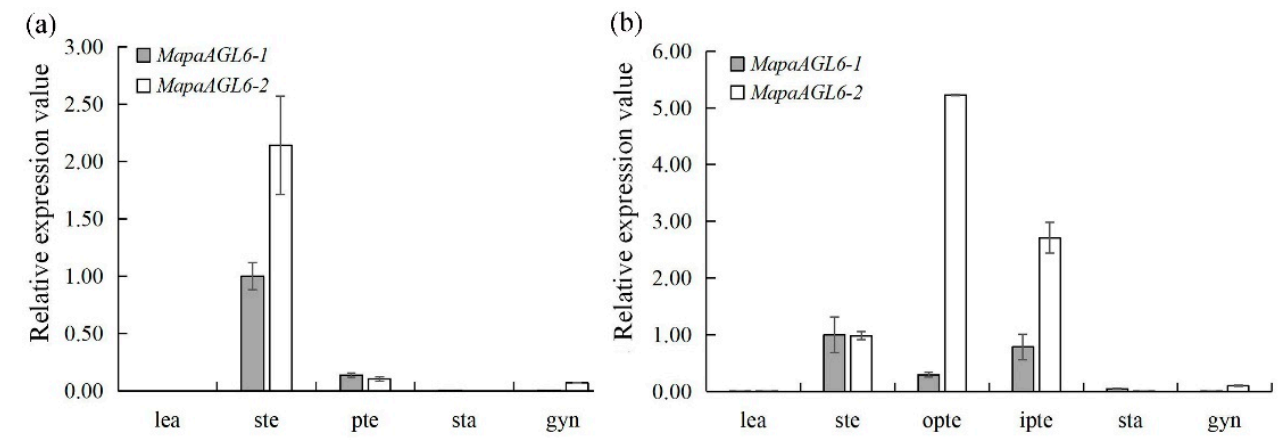

Figure 4. Expression of MapaAGL6-1 and MapaAGL6-2 in the juvenile leaves (lea), sepaloid tepals (ste), petaloid tepals (pte), outer petaloid tepals (opte), inner petaloid tepals (ipte), stamens (sta), and and gynoecia (gyn) detected by qPCR with $M$. patungensis actin as the normalization. (a) Expression of MapaAGL6-1 and MapaAGL6-2 in different floral organs of $27 \mathrm{~mm}$ long floral buds and juvenile leaves, respectively; (b) expression of MapaAGL6-1 and MapaAGL6-2 in different floral organs of $40 \mathrm{~mm}$ long floral buds and juvenile leaves, respectively.

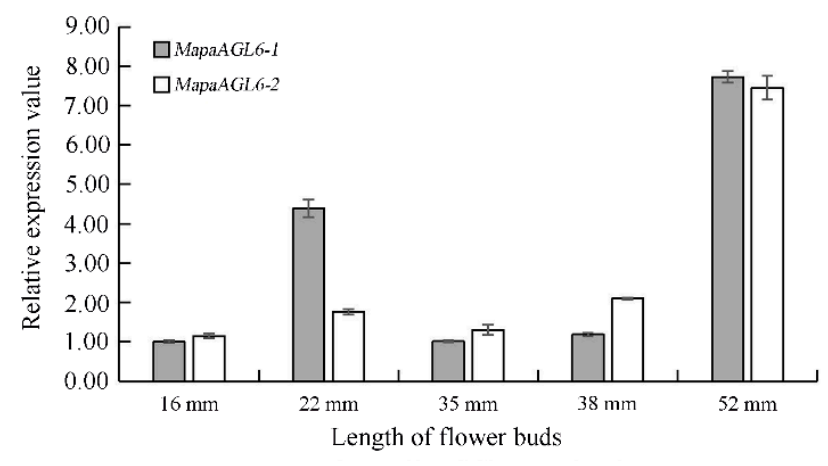

Figure 5. Relative expression of MapaAGL6-1 and MapaAGL6-2 in floral buds of different lengths.

\subsection{Ectopic Expression of MapaAGL6-1 and MapaAGL6-2 in ap1-10 Arabidopsis}

In order to explore their roles in regulating floral development, we transformed 35S::MapaAGL6-1 and 35S::MapaAGL6-2 constructs into Arabidopsis ap1-10 mutants that produced flowers with petal loss and abnormal sepals to create complementation lines in these well-characterized mutant backgrounds, respectively. After identification by PCR and quantitative real time PCR (Figure 6), thirty 35S::MapaAGL6-1 and forty 35S::MapaAGL6-2 of independent homozygous transformants under ap1-10 mutant background were obtained, respectively. Moreover, twenty ap1-10 homozygous transformants with the pBI121 vector only were also obtained. 

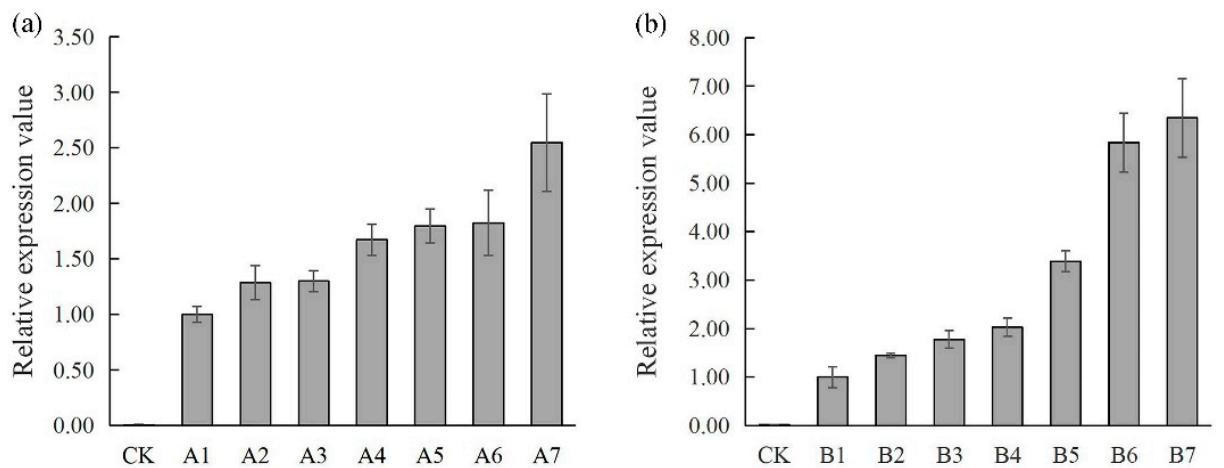

Figure 6. The 35S::MapaAGL6-1 and 35S::MapaAGL6-2 independent homozygous transformants under Arabidopsis ap1-10 mutant background identified by qPCR, respectively. (a) The relative expression of MapaAGL6-1 was detected in the homozygous ap1-10 mutant Arabidopsis; (b): Expression of MapaAGL6-2 was confirmed in the homozygous ap1-10 mutant Arabidopsis. (CK) Homozygous ap1-10 mutant Arabidopsis; (A1) 35S::MapaAGL6-1 transgenic line without phenotypic change; (A2, A3, and A4) 35S::MapaAGL6-1 transgenic lines displayed medium rescue phenotypes; (A5, A6, and A7) 35S::MapaAGL6-1 transgenic lines showed strong complementation phenotypes; (B1, B2, B3, and B4) 35S::MapaAGL6-2 transgenic lines without phenotypic change; (B5, B6, and B7) 35S::MapaAGL6-2 transgenic lines displayed phenotypic changes in floral structure.

Among a total of 30 35S::MapaAGL6-1 homozygous ap1-10 transformants, 16 (53.33\%) lines showed strong complementation phenotypes with flowers consisting of two, three, or four sepals in the first whorl, three petals in whorl 2, and normal reproductive structure (stamens and gynoecia) in the inner two whorls (Figure 7e-g). Four (13.33\%) lines displayed medium rescue of flower phenotypes. Moreover, half of them produced flowers with two or three sepals in the first whorl, one or two petals in whorl 2, and normal stamens and gynoecia in whorl 3 and whorl 4, respectively (Figure 7h). One line has many cluster bract-like sepals enclosing normal reproductive organs (six stamens and a superior gynoecium) on an obvious compression floral axis (Figure 7i). One line produces flowers without petals (Figure 7j,k). Ten (33.33\%) lines displayed no obvious changes in floral phenotypes. Among a total of 40 35S::MapaAGL6-2 homozygous ap1-10 transformants, 22 (55.00\%) lines displayed phenotypic changes in floral structure. They produced a petaloid structure on the top of the filament (Figure 7l,m) or had one petal in the second whorl (Figure 7n). Eighteen $(45.00 \%)$ lines displayed no obvious changes in floral phenotypes. In addition, the daughter lines generated from a cross between 35S::MapaAGL6-1 transgenic plants showing strong phenotypes and 35S::MapaAGL6-2 transgenic plants showing phenotypic changes could produce the normal inflorescence and perfect flowers consisting of four whorls of floral organs (Figure 7o,p). However, all transformants with the pBI121 vector only mimicked the flower phenotypes of Arabidopsis ap1-10 mutants. 


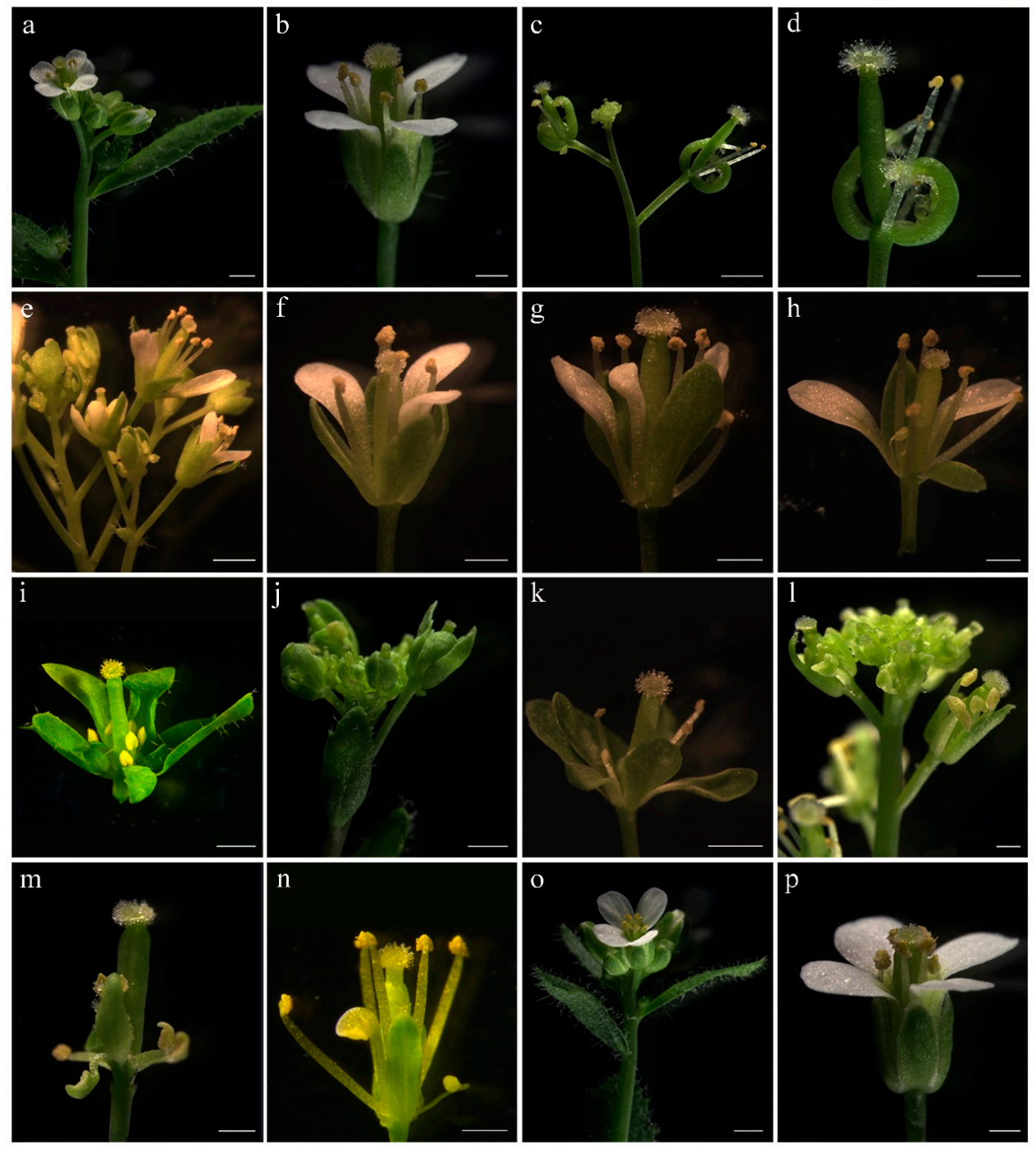

Figure 7. Phenotype comparison of wild-type, homozygous ap1-10 mutant, 35S::MapaAGL6-1, and 35S::MapaAGL6-2 transgenic Arabidopsis under a homozygous ap1-10 mutant background, respectively. (a) Inflorescence of wild-type Arabidopsis; (b) wild-type Arabidopsis flower with normal four whorls floral organs; (c) inflorescence of homozygous ap1-10 mutant Arabidopsis; (d) flower of homozygous ap1-10 mutant Arabidopsis with first whorl sepal converted into carpel-like organs bearing ovules and numbers of stamens reduced; (e-k) inflorescence or flowers of 35S::MapaAGL6-1 transgenic Arabidopsis under homozygous ap1-10 mutants with different degrees of rescue: (e) inflorescence of 35S::MapaAGL6-1 transgenic Arabidopsis displaying strong complementation phenotypes; (f) flowers of a rescued ap1-10 mutant with four sepals in whorl 1, three petals in whorl 2, and normal stamens and gynoecia in whorl 3 and whorl 4, respectively; (g) flowers of a rescued ap1-10 mutant with two sepals in the first whorl, three petals in the second whorl, and normal stamens and gynoecia in the inner two whorls; (h) flowers of a rescued ap1-10 mutant with two sepals in whorl 1, two petals in whorl 2, six stamens in whorl 3, and normal gynoecia in whorl 4; (i) cluster bract-like sepals enclosing normal reproductive structures on an obvious compression floral axis; $(\mathbf{j}-\mathbf{k})$ inflorescence and flowers of a rescued ap1-10 mutant without petal whorl; (1-n) inflorescence or flowers of 35S::MapaAGL6-2 transgenic Arabidopsis under homozygous ap1-10 mutants with different degrees of rescue: (1-m) inflorescence and flowers of a rescued ap1-10 mutant with petaloid anthers on the top of the filament; (n) flowers of a rescued ap1-10 mutant with one petal in the second whorl; $(\mathbf{o}-\mathbf{p})$ inflorescence and flowers of the daughter lines generated from a cross between 35S::MapaAGL6-1 transgenic plants showing strong phenotypes and 35S::MapaAGL6-2 transgenic plants showing phenotypic changes under homozygous ap1-10 mutant background. Bars: (b,d,f-h,l-n,p) $500 \mu \mathrm{m}$; (a,c,e,i-k,o) $1 \mathrm{~mm}$. 


\section{Discussion}

Perianth is widely present in almost all extant angiosperms and a double perianth with sepals and petals differentiated is considered to be a key innovation for eudicots [29]. Although the molecular developmental basis for perianth differentiation in eudicots model species, such as Arabidopsis and Antirrhinum, is well explained by the ABCE-model [3,30], how perianth identity is programmed in magnoliids remains unclear. The perianth of most magnoliids species consists of two or three whorls of morphologically identical laminar organs, termed tepals [31], and hence exhibits a considerable difference from eudicot perianths, which consist of morphologically distinct sepals and petal whorls. Moreover, the tepals in basal angiosperms show more similar traits with the sepal in eudicots except their showy color, which indicates that the tepal identity program may be more different with perianth form eudicots.

AfimAGL6, an AGL6 ortholog from magnoliids species A. fimbriata, is only expressed in flowers and capsules, and is involved in regulating petaloid sepal (tepal) development [10]. In another magnoliids species, C. praecox, the AGL6 ortholog is mainly expressed in tepals and carpels but is lowly expressed in stamen and juvenile leaves and shows a potential role in controlling flowering time and floral organ identity [20]. Moreover, the AGL6-like gene of M. grandiflora is expressed only in tepals and an AGL6 ortholog, AmtrAGL6 from A. trichopoda, is highly expressed in tepals but is weakly expressed in other floral organs [6,8]. If function can be inferred from expression, AGL6 orthologs are reasonable candidates for A-function genes in basal angiosperms. In addition, an AGL6-like gene, NdAGL6 from a basal eudicot species $N$. damascena, has been recently identified as conferring A-function [7]. However, AGL6 orthologs from asterid eudicots show broader expression zones and play diverse roles in regulating floral development. For examples, tomato SlAGL6 and Petunia PhAGL6 have been seen to be expressed in four floral organs. SlAGL6 plays a key role in regulating tomato sepal and petal development, while PhAGL6 plays a key role in regulating petal, anther, and ovary development $[15,16]$. In monocots, the rice AGL6 ortholog, OsMADS6, has been found to be strongly expressed in all floral organs and has been observed to be involved in regulating flower development of four whorls [11,12]. All these data suggest the functional evolution of AGL6 orthologs follows their changes in expression pattern during flower evolution of angiosperms.

In M. patungensis, MapaAGL6-1 is only expressed in the perianth whorls, and MapaAGL6-2 is strongly expressed in the perianth whorls but is lowly expressed in gynoecium. Moreover, with petaloid tepal development, both gene expressions are expanded into the petaloid tepal whorls and achieve a high level in this region, which suggests that they may be essential for the perianth identity. In addition, the function of $A G L 6$ orthologs from orchids can be inferred from their expressions. For examples, CgAGL6-1 from $C$. goeringii has been found to be strongly expressed in the sepals and petals and has been seen to be involved in promoting sepal/petal formation, while $C g A G L 6-3$ from C. goeringii has been found to be highly expressed in the lips and to be involved in lip development [14]. This also provides a clue to understanding the subfunctionalization of $A G L 6$ orthologs following gene duplication events. In M. patungensis, ectopic expression of MapaAGL6-1 results in strong complementation phenotypes in the Arabidopsis ap1-10 flower and produces normal flowers consisting of the normal four whorls of floral organs, with only the petal number reduced in whorl 3 (Figure 7e,f); ectopic expression of MapaAGL6-2 in Arabidopsis ap1-10 mutant only results in petals partly rescued in whorl 2 but fails to terminate carpelloid organs development in whorl 1 (Figure $7 \mathrm{~m}, \mathrm{n}$ ). In addition, the daughter lines generated from a cross between 35S::MapaAGL6-1 transgenic plants showing strong phenotypes and 35S::MapaAGL6-2 transgenic plants showing phenotypic changes could produce normal inflorescence and perfect flowers consisting of four whorls of floral organs. All these data raise the possibility that MapaAGL6-1 contributes to A-function and MapaAGL6-2 involves petaloid tepal development in M. patungensis. Our study also provides a clue to the development and evolution of differentiated perianth in basal angiosperms. 
Supplementary Materials: Supplementary materials can be found at http://www.mdpi.com/1999-4907/10/8/669/s1. Table S1: Information on Sequences selected for alignments and phylogenetic analyses from NCBI GenBank.

Author Contributions: Z.L. designed the experiments and wrote the manuscript. K.Z., L.L., and Y.F. conducted the experiments and data analysis. F.C. discussed the results and revised the manuscript. All authors read and approved the final manuscript.

Funding: This work was supported by a grant from the National Natural Science Foundation of China (grant no. 31570651), and the Open Research Fund from Key Laboratory of Three Gorges Regional Plant Genetics \& Germplasm Enhancement (2016KBC01).

Conflicts of Interest: The authors declare no conflict of interest.

\section{Abbreviations}

$\begin{array}{ll}\text { LSD } & \text { Least Significant Difference } \\ \text { ORF } & \text { Open Reading Frame } \\ \text { PCR } & \text { Polymerase Chain Reaction } \\ \text { qPCR } & \text { Quantitative real-time PCR }\end{array}$

\section{References}

1. Coen, E.S.; Meyerowitz, E.M. The war of the whorls: Genetic interactions controlling flower development. Nature 1991, 353, 31-37. [CrossRef] [PubMed]

2. Pelaz, S.; Ditta, G.S.; Baumann, E.; Wisman, E.; Yanofsky, M.F. B and C floral organ identity functions require SEPALLATA MADS-box genes. Nature 2000, 405, 200-203. [CrossRef] [PubMed]

3. Krizek, B.A.; Fletcher, J.C. Molecular mechanisms of flower development: An armchair guide. Nat. Rev. Genet. 2005, 6, 688-698. [CrossRef] [PubMed]

4. Zahn, L.M.; Feng, B.M.; Ma, H. Beyond the ABC-Model: Regulation of floral homeotic genes. Adv. Bot. Res. 2006, 44, 164-196.

5. Davies, B.; Cartolano, M.; Schwarz-Sommer, Z. Flower development: The Antirrhinum perspective. Adv. Bot. Res. 2006, 44, 279-320.

6. Soltis, P.S.; Soltis, D.E.; Kim, S.; Chanderbali, A.; Buzgo, M. Expression of floral regulators in basal angiosperms and the origin and evolution of ABC function. Adv. Bot. Res. 2006, 44, 483-506.

7. Wang, P.; Liao, H.; Zhang, W.; Yu, X.; Zhang, R.; Shan, H.; Duan, X.; Yao, X.; Kong, H. Flexibility in the structure of spiral flowers and its underlying mechanisms. Nat. Plants 2015, 2, 15188. [CrossRef] [PubMed]

8. Kim, S.; Koh, J.; Yoo, M.J.; Kong, H.; Hu, Y.; Ma, H.; Soltis, P.S.; Soltis, D.E. Expression of floral MADS-box genes in basal angiosperms: Implications for the evolution of floral regulators. Plant J. 2005, 43, 724-744. [CrossRef] [PubMed]

9. Pabón-Mora, N.; Suárez-Baron, H.; Ambrose, B.A.; González, F. Flower Development and Perianth Identity Candidate Genes in the Basal Angiosperm Aristolochia fimbriata (Piperales: Aristolochiaceae). Front. Plant Sci. 2015, 6, 1095. [CrossRef]

10. Peréz-Mesa, P.; Suárez-Baron, H.; Ambrose, B.A.; González, F.; Pabón-Mora, N. Floral MADS-box protein interactions in the early diverging angiosperm Aristolochia fimbriata Cham. (Aristolochiaceae: Piperales). Evol. Dev. 2019, 21, 96-110. [CrossRef]

11. Li, H.; Liang, W.; Jia, R.; Yin, C.; Zong, J.; Kong, H.; Zhang, D. The AGL6-like gene OsMADS6 regulates floral organ and meristem identities in rice. Cell Res. 2010, 20, 299-313. [CrossRef] [PubMed]

12. Tao, J.; Liang, W.; An, G.; Zhang, D. OsMADS6 Controls Flower Development by Activating Rice FACTOR OF DNA METHYLATION LIKE1. Plant Physiol. 2018, 177, 713-727. [CrossRef] [PubMed]

13. Dirks-Mulder, A.; Butôt, R.; van Schaik, P.; Wijnands, J.W.; van den Berg, R.; Krol, L.; Doebar, S.; van Kooperen, K.; de Boer, H.; Kramer, E.M.; et al. Exploring the evolutionary origin of floral organs of Erycina pusilla, an emerging orchid model system. BMC Evol. Biol. 2017, 17, 89. [CrossRef] [PubMed]

14. Xiang, L.; Chen, Y.; Chen, L.; Fu, X.; Zhao, K.; Zhang, J.; Sun, C. B and E MADS-box genes determine the perianth formation in Cymbidium goeringii Rchb.f. Physiol. Plant. 2018, 162, 353-369. [CrossRef] [PubMed]

15. Yu, X.; Chen, G.; Guo, X.; Lu, Y.; Zhang, J.; Hu, J.; Tian, S.; Hu, Z. Silencing SlAGL6, a tomato AGAMOUS-LIKE6 lineage gene, generates fused sepal and green petal. Plant Cell Rep. 2017, 36, 959-969. [CrossRef] [PubMed] 
16. Rijpkema, A.S.; Zethof, J.; Gerats, T.; Vandenbussche, M. The petunia AGL6 gene has a SEPALLATA-like function in floral patterning. Plant J. 2009, 60, 1-9. [CrossRef] [PubMed]

17. Sauquet, H.; von Balthazar, M.; Magallón, S.; Doyle, J.A.; Endress, P.K.; Bailes, E.J.; Barroso de Morais, E.; Bull-Hereñu, K.; Carrive, L.; Chartier, M.; et al. The ancestral flower of angiosperms and its early diversification. Nat. Commun. 2017, 8, 16047. [CrossRef] [PubMed]

18. Tamura, K.; Peterson, D.; Peterson, N.; Stecher, G.; Nei, M.; Kumar, S. MEGA5: Molecular evolutionary genetics analysis using maximum likelihood, evolutionary distance, and maximum parsimony methods. Mol. Biol. Evol. 2011, 28, 2731-2739. [CrossRef] [PubMed]

19. Kim, S.; Soltis, P.S.; Soltis, D.E. AGL6-like MADS-box genes are sister to AGL2-like MADS-box genes. J. Plant Biol. 2013, 56, 315-325. [CrossRef]

20. Wang, B.G.; Zhang, Q.; Wang, L.G.; Duan, K.; Pan, A.H.; Tang, X.M.; Sui, S.Z.; Li, M.Y. The AGL6-like Gene CpAGL6, a Potential Regulator of Floral Time and Organ Identity in Wintersweet (Chimonanthus praecox). J. Plant Growth Regul. 2011, 30, 343-352. [CrossRef]

21. Klap, C.; Yeshayahou, E.; Bolger, A.M.; Arazi, T.; Gupta, S.K.; Shabtai, S.; Usadel, B.; Salts, Y.; Barg, R. Tomato facultative parthenocarpy results from SIAGAMOUS-LIKE 6 loss of function. Plant Biotechnol. J. 2017, 15, 634-647. [CrossRef] [PubMed]

22. Ohmori, S.; Kimizu, M.; Sugita, M.; Miyao, A.; Hirochika, H.; Uchida, E.; Nagato, Y.; Yoshida, H. MOSAIC FLORAL ORGANS1, an AGL6-like MADS box gene, regulates floral organ identity and meristem fate in rice. Plant Cell 2009, 21, 3008-3025. [CrossRef] [PubMed]

23. Zhang, B.; Liu, Z.X.; Ma, J.; Song, Y.; Chen, F.J. Alternative splicing of the AGAMOUS orthologous gene in double flower of Magnolia stellata (Magnoliaceae). Plant Sci. 2015, 241, 277-285. [CrossRef] [PubMed]

24. Clough, S.J.; Bent, A.F. Floral dip: A simplified method for Agrobacterium-mediated transformation of Arabidopsis thaliana. Plant J. 1998, 16, 735-743. [CrossRef] [PubMed]

25. Li, L.Y.; Fang, Z.W.; Li, X.F.; Liu, Z.X. Isolation and Characterization of the C-class MADS-box Gene from the Distylous Pseudo-cereal Fagopyrum esculentum. J. Plant Biol. 2017, 60, 189-198. [CrossRef]

26. Riechmann, J.L.; Krizek, B.A.; Meyerowitz, E.M. Dimerization specificity of Arabidopsis MADS domain homeotic proteins APETALA1, APETALA3, PISTILLATA, and AGAMOUS. Proc. Natl. Acad. Sci. USA 1996, 93, 4793-4798. [CrossRef]

27. Yang, Y.; Jack, T. Defining subdomains of the K domain important for protein-protein interactions of plant MADS proteins. Plant Mol. Biol. 2004, 55, 45-59. [CrossRef]

28. Sun, W.; Huang, W.; Li, Z.; Song, C.; Liu, D.; Liu, Y.; Hayward, A.; Liu, Y.; Huang, H.; Wang, Y. Functional and evolutionary analysis of the AP1/SEP/AGL6 superclade of MADS-box genes in the basal eudicot Epimedium sagittatum. Ann. Bot. 2014, 113, 653-668. [CrossRef]

29. Endress, P.K. Angiosperm Floral Evolution: Morphological Developmental Framework. Adv. Bot. Res. 2006, 44, 1-61.

30. Monniaux, M.; Vandenbussche, M. How to Evolve a Perianth: A Review of Cadastral Mechanisms for Perianth Identity. Front. Plant. Sci. 2018, 9, 1573. [CrossRef]

31. Endress, P.K. Evolutionary diversification of the flowers in angiosperms. Am. J. Bot. 2011, 98, 370-396. [CrossRef] [PubMed]

(C) 2019 by the authors. Licensee MDPI, Basel, Switzerland. This article is an open access article distributed under the terms and conditions of the Creative Commons Attribution (CC BY) license (http://creativecommons.org/licenses/by/4.0/). 